\title{
Haptic Search for Spheres and Cubes
}

\author{
Myrthe A. Plaisier, Wouter M. Bergmann Tiest, and Astrid M.L. Kappers \\ Helmholtz Institute, Utrecht University, The Netherlands \\ M.A.Plaisier@phys.uu.nl
}

\begin{abstract}
If you have multiple objects in your pocket, some are easy to find among the other ones, for instance, when they differ much in material properties or shape. Information on which haptic features stand out among others is valuable for research into the haptic system in general, but also for haptic interface design. In this research we focussed on saliency of shape, by letting subjects search for cubes or spheres. Response times were measured as a function of the number of items. We found that search for a cube among spheres is more efficient than search for a sphere among cubes and that the dynamics of the sliding of the shapes along each other play an important role in haptic search.
\end{abstract}

Keywords: Haptic search, Psychophysics, Response times.

\section{Introduction}

Keys, some loose change, a mobile phone and a handkerchief are all examples of objects that might be in your pocket at any given time. Several times a day we reach into our pocket to take out one of these items. Some of these items are very difficult to find and sometimes we resort to taking out the whole content of our pocket to visually find what we were looking for. Other items are remarkably easy to find using only the haptic modality. For instance, when the item you want to find differs much in size from the other items or is made out of a material that is perceived as very different from the rest. Besides relative size and material properties, shape can provide an important cue for finding a specific item. So what makes some objects stand out among others? This is an important question, because it gives insight into how haptic object recognition takes place and thereby provides important information on the haptic system. On the other hand, knowing which haptic features are detected fast and accurately can also be of value for haptic interface design. For instance, to render easy recognizable virtual haptic objects.

In vision, object saliency has often been addressed using search tasks [1]. Usually varying numbers of items are displayed on a screen and subjects are instructed to respond as fast as possible whether a certain target item is present among the other items. Response times are then measured as a function of the number of items. The slope of a straight line through the points indicates the extra search time needed per extra item and is a measure for how much a target item stands out among other (distractor) items. The search paradigm has been proven to be very useful in vision and can be extended to the haptic domain.

M. Ferre (Ed.): EuroHaptics 2008, LNCS 5024, pp. 275 2822008

(C) Springer-Verlag Berlin Heidelberg 2008 
It has been shown that the haptic system is quite accurate when it comes to recognition of familiar objects [2. Object recognition often can even be done through only brief contact with the object, which has been labeled a 'haptic glance' 3. It is probable that there are primitive features that can be extracted relatively fast by the haptic system to enable such fast object recognition. So what are these features? Lederman and Klatzky looked into this problem using a haptic search task. In their setup, varying numbers of items were pressed onto the subjects' fingers 4]. Consequently, exploratory movements were constrained to small finger movements. They found that especially search for material properties such as roughness was efficient, but also search for an edge among no-edge items was relatively fast.

Most research on haptic shape perception has been concerned with curvature perception. When exploring objects with sizes that can be easily held in hand, the pressure profile on the skin and the cutaneous signals that arise are important. It has been shown that subjects can discriminate curved surfaces pressed to their finger pads using cutaneous information only [5]6]. Furthermore, subjects can estimate the orientation of a cylinder pressed to the finger pad fairly well [7].

In daily life objects are not normally pressed onto the finger pads. In fact, we usually hold multiple objects in our hand and we can freely rearrange these objects. Besides the pressure profile on the skin, also the dynamics of the different objects in terms of, for instance, how easy they slide along each other determine how easy a certain object is found. We set out to investigate haptic search under free exploration when grasping several objects, differing in shape, in the hand. Because curvature and edges are important features for haptic shape perception we used spheres, that have a curved surface without edges, and cubes that do have edges. Subjects had to search for a sphere among cubes or a cube among spheres. Response times were then recorded as a function of the number of items. The slope of this function indicates the amount of extra time needed per item and thereby is a measure of how efficient a search was performed. If this is the same for the two conditions in our experiment, then this indicates that curvature and edges are processed equally fast. To enable accurate response time measurements, a stand alone device with a touch sensitive contact was custom made.

\section{Experimental Design and Setup}

\subsection{Participants}

Eight paid subjects (5 male, mean age 22) participated in the experiments. All subjects were right-handed according to Coren's test [8] and none of them reported any known hand deficits.

\subsection{Stimuli and Setup}

Stimuli consisted of spheres and cubes made out of brass. Both shapes had the same volume and, consequently, the same mass. This ensured that there were 
no weight cues. The size of the shapes was chosen such that varying numbers of the items could fit comfortably in the subject's hand and would easily be manipulated. The spheres had a diameter of $1.9 \mathrm{~cm}$ and the cubes had edge lengths of $1.5 \mathrm{~cm}$. The objects were suspended from electrical wires, which could be plugged into the touch sensitive contact of the response time measuring device.

Response times were recorded using a custom build stand-alone response time measuring device. The flowchart of this device is shown in Fig. 1. The moment of contact with the stimulus was registered through a touch sensitive contact and response time measurement was started. Inside the device, a $50 \mathrm{~Hz}$ electrical field was generated. When the contact was touched the impedance change caused a drop in the field frequency. If this change reached a threshold value the time measurement was started. This way, only one contact point was needed. Furthermore, time measurement started when the touch-sensitive contact was actually touched and not already upon approaching it. Time measurement was terminated with a vocal response registered through a headset microphone. The response time was then fed into a computer where it was stored. This enabled accurate response time measurements, because it started at the moment the stimulus was touched, while the subjects initialized the start of the trial themselves. Therefore, the beginning and ending of a trial were induced through quite natural actions.

\subsection{Experimental Design}

Subjects were blindfolded during the experiments. Because there were no noticeable sound differences between target present and target absent trials, there was no need for the subjects to wear earplugs. To be certain of optimal performance, subjects were instructed to use their dominant hand. Before a trial was started, they placed their hand palm up in a holder (Fig. 2). They were instructed to reach upwards and grab all items simultaneously and respond as fast as possible if a target item was present (by calling out the Dutch equivalents of 'yes' and 'no'). It was also emphasized that the answer should be correct. During trials they were allowed to release items from their hand, but they were instructed to only do this when they thought this would enhance the efficiency of their performance. There were no restrictions on hand movements.

The experiment consisted of two conditions. In the first condition a sphere was the target item and cubes were the distractor items. For the other condition target and distractor identities were interchanged and the target item was a cube while the distractor items were spheres. In both conditions the number of items was varied and the subjects were presented with a total of 3, 4, 5, 6 or 7 items. The different conditions were performed in separate blocks of trials and each block took approximately 45 minutes. Subjects were informed that they could ask for a five minute break if they felt they were getting tired, but none of them did. Subjects were informed of which shape would be the target item. All subjects participated in both conditions and the order in which the conditions were performed was roughly counter-balanced over subjects. In each condition they performed 20 trials for each number of items and in half of the trials a target 


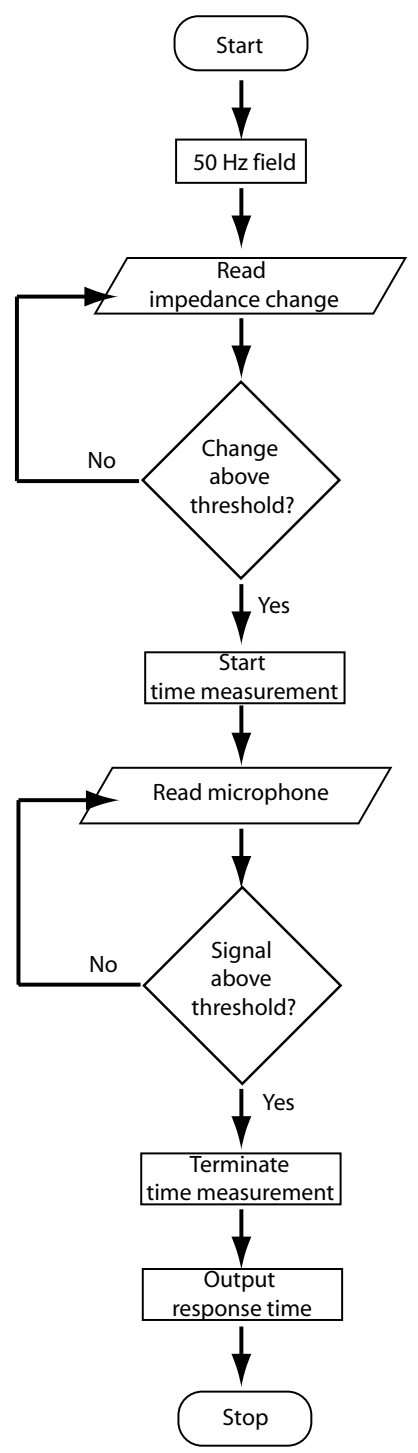

Fig. 1. Flowchart of the stand-alone response time measuring device. Time measurement was started when a touch-sensitive contact was touched and it was terminated with a vocal response.

item was present. For each trial a new set of stimuli was suspended. The blocks of trials were preceded by a minimum of 20 practice trials until 10 in a row were performed correctly. For none of the subjects did the training session exceed 30 trials. During training as well as during the actual experiment subjects received feedback on whether their answer was correct. When an incorrect response was given, the trial was repeated at the end of the block. 

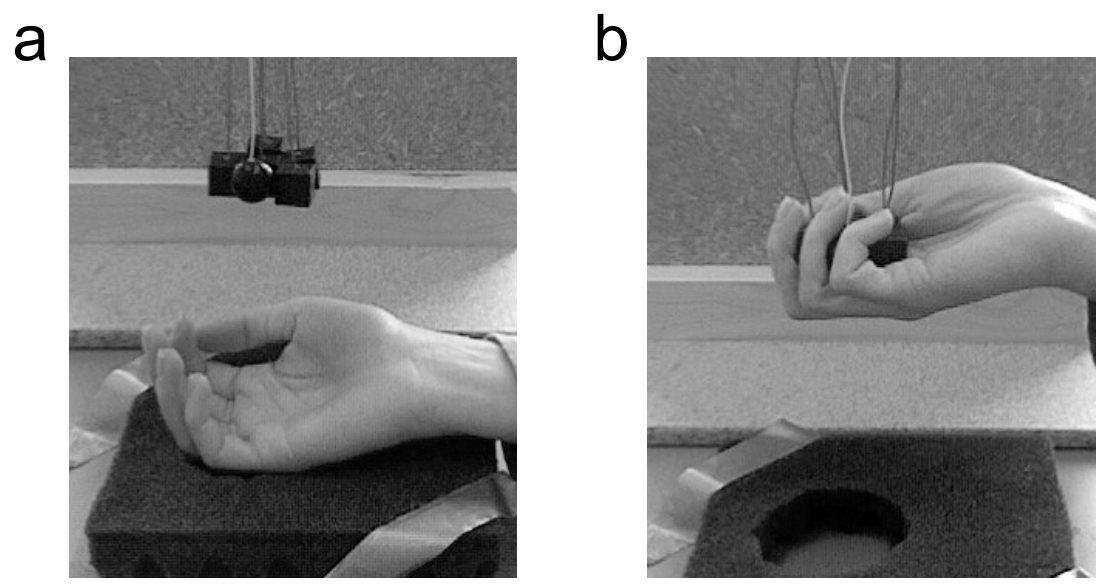

Fig. 2. Picture of the setup. a) The subject's hand was placed in the holder below the stimulus. In this case the stimulus consisted of a sphere among cubes. b) The subject reached upwards and grasped all items simultaneously. Response time measurement was started upon physical contact with the stimulus.

\section{Results}

Fig. 3 shows the response times as a function of the number of items averaged over all subjects. In Fig. 3a target present trials for both conditions are presented, while the target absent trials are shown in Fig. 3b. Error rates were below $1.5 \%$ in each condition. The maximum number of trials that had to be repeated in one block of trials was three, so increased exposure to the experiment due to repetition of trials was negligible. It can be seen that for both conditions the slope for the target absent trials was higher than for the target present trials. This is commonly found in search tasks in both the visual and the haptic domain. The slope for the sphere among cubes was higher than for the cube among spheres for target present as well as target absent trials. In the target present case linear regression yielded a slope of $70 \pm 7 \mathrm{~ms} /$ item (SE) for the cube among spheres and $112 \pm 20 \mathrm{~ms} /$ item for the sphere among cubes. For the target absent trials these slopes were $180 \pm 10 \mathrm{~ms} /$ item and $533 \pm 20 \mathrm{~ms} /$ item, respectively.

Linear regression was also performed on the single subject data for target present and absent trials in both conditions $\left(R^{2} \geq 0.78\right)$. A 2 by 2 (condition $\times$ target presence) Analysis Of Variance (ANOVA) was performed on the slopes resulting from the linear regression. This showed significant main effects for condition $\left(F_{1,7}=21.2, p=0.002\right)$ as well as target presence $\left(F_{1,7}=32.9, p=0.001\right)$ and the interaction term $\left(F_{1,7}=14.9, p=0.006\right)$. The significant interaction term indicates that the slope difference between target present and absent trials depended on the condition. When distractors were spheres the ratio between the slopes for target present and absent trials was approximately 2.5 while when the distractors were cubes this ratio was almost 5 . 

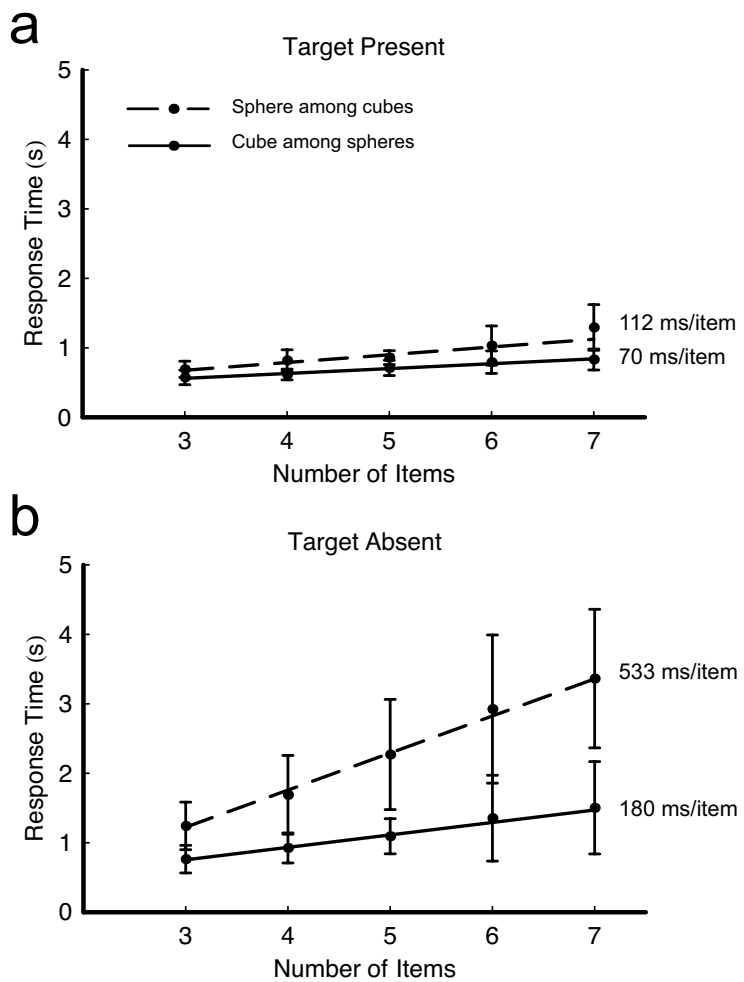

Fig. 3. Response times, averaged over subjects, as a function of the number of items. The error bars indicate the standard error. The solid line represents linear regression to the cube among spheres condition, while the dashed line represents linear regression to the sphere among cubes condition. The slopes resulting from the regression are indicated in the graphs. a) Target present trials and b) target absent trials.

\section{Discussion and Conclusions}

Our results show that haptic search for a cube among spheres is significantly different from search for a sphere among cubes. Note that the only difference between these conditions was reversing target and distractor identities and such differences are usually referred to as search asymmetries. The search slope for a sphere among cubes was larger than for a cube among spheres indicating that the former was more difficult. This indicates that it is easier to find an item with edges among items without edges than the reversed case. Another part of the explanation could be related to the fact that it is easier to manipulate a hand full of spheres than a hand full of cubes. The mutual contact area between two cubes is much larger than for two spheres; consequently friction is much higher when rearranging a hand full of cubes. 
There was also a large difference in the ratios between target present and absent slopes between the two conditions. This was also indicated by the significant interaction term in the statistical analysis. The large slope for the target absent trials in the sphere among cubes condition suggests that subjects were more uncertain on whether there was a target item than in the cube among spheres condition, while in the target present trials the target was found relatively fast. Mixed cubes and spheres do not stack very well and when squeezed. the target cube or sphere will probably slide out from between the distractors. In target absent trials, when no target slid out, subjects had to search the items more elaborately to be certain there was no target item. The results suggest that subjects could use this cue. During training trials they could already learn to use this cue making it unlikely that there was a learning effect during the actual experiment. The large target absent search slopes when cubes were used as distractors suggest that cubes were more difficult to manipulate than spheres.

Extensive research of visual search tasks has led to various models that usually distinguish between parallel and serial search 910. In a serial self terminating search, all items are visited subsequently. The ratio between target present and absent search slopes would then be $1: 2$, because in target present trials on average only half of the items has to be visited before the target is found, while in target absent trials all items have to be visited. If this ratio is larger than $1: 2$, search is more efficient than purely serial and several items are processed simultaneously (parallel search). On the other hand, ratios much smaller than $1: 2$, like $1: 2.5$ and $1: 5$ as found in the present experiment, are not commonly found in visual search tasks. A smaller ratio could be explained by subjects visiting items several times instead of all items once.

An important difference between the present research and previous visual and haptic search tasks is that subjects could freely manipulate and rearrange the items in our design. This means that the dynamics of the shapes when sliding along each other when grasped and manipulated by the subjects play a role in the present research. This factor has never been present in previous research involving search tasks in both the visual and the haptic domain.

In daily life, haptic search often involves manipulating three-dimensional objects in our hand. By presenting subjects with three-dimensional items held in their hand we investigated haptic search under conditions that resemble haptic search in daily life situations. Not only do our results show that edges are detected relatively fast, they show that the dynamics of sliding shapes can be an important factor in haptic search tasks. For the design of haptic interfaces in which operation must be fast and accurate, haptic saliency of certain shapes might be used to make operation more efficient. Research such as presented here, provides information on which shape features are important for efficient shape recognition. This type of 'primitive' features could be used to render virtual haptic objects that can be easily recognized by the user.

Acknowledgments. This research was supported by a grant from the Netherlands Organisation for Scientific Research. 


\section{References}

1. Treisman, A., Gormican, S.: Feature analysis in early vision: evidence from search asymmetries. Psychological Review 95, 15-48 (1988)

2. Klatzky, R.L., Lederman, S.J., Metzger, V.A.: Identifying objects by touch: an "expert system". Perception and Psychophysics 37, 299-302 (1985)

3. Klatzky, R.L., Lederman, S.J.: Identifying objects from a haptic glance. Perception \& Psychophysics 57, 1111-1123 (1995)

4. Lederman, S.J., Klatzky, R.L.: Relative availability of surface and object properties during early haptic processing. Journal of Experimental Psychology: Human Perception and Performance 23, 1680-1707 (1997)

5. Goodwin, A.W., John, K.T., Marceglia, A.H.: Tactile discrimination of curvature by humans using only cutaneous information fron the fingerpads. Experimental Brain Research 86, 663-672 (1991)

6. Jenmalm, P., Birznieks, I., Goodwin, A.W., Johansson, R.S.: Influence of object shape on responses of human tactile afferents under conditions characteristic of manipulation. European Journal of Neuroscience 18, 164-176 (2003)

7. Dodson, M.J., Goodwin, A.W., Browning, A.S., Gehring, H.M.: Peripheral neural mechanisms determining the orientation of cylinders grasped by the digits. Journal of Neuroscience 18, 521-530 (1998)

8. Coren, S.: The left-hander syndrome: The causes and consequences of lefthandedness. Vintage Books, New York (1993)

9. Treisman, A., Gelade, G.: A feature-integration theory of attention. Cognitive Psychology 12, 97-136 (1980)

10. Wolfe, J.M., Cave, K.R., Franzel, S.L.: Guided search: an alternative to the feature integration model for visual search. Journal of Experimental Psychology: Human Perception and Performance 15, 419-433 (1989) 